\title{
Blood parasites in vectors reveal a united blackfly community in the upper canopy
}

\author{
Nayden Chakarov ${ }^{1,2^{*}}$, Helge Kampen ${ }^{3}$, Anja Wiegmann ${ }^{1}$, Doreen Werner ${ }^{4}$ and Staffan Bensch ${ }^{2}$
}

\begin{abstract}
Background: The behaviour of blood-sucking arthropods is a crucial determinant of blood protozoan distribution and hence of host-parasite coevolution, but it is very challenging to study in the wild. The molecular identification of parasite lineages in vectors can be a useful key to understand the behaviour and transmission patterns realised by these vectors.

Methods: In this study, we collected blackflies around nests of three raptor species in the upper forest canopy in central Europe and examined the presence of vertebrate DNA and haemosporidian parasites in them. We molecularly analysed 156 blackfly individuals, their vertebrate blood meals, and the haemosporidian parasite lineages they carried.

Results: We identified nine species of Simulium blackflies, largely belonging to the subgenera Nevermannia and Eusimulium. Only $1 \%$ of the collected specimens was visibly engorged, and only $4 \%$ contained remains of host DNA. However, in 29\% of the blackflies Leucocytozoon lineages were identified, which is evidence of a previous blood meal on an avian host. Based on the known vertebrate hosts of the recorded Leucocytozoon lineages, we can infer that large and/or abundant birds, such as thrushes, crows, pigeons, birds of prey, owls and tits are the main targets of ornithophilic blackflies in the canopy. Blackfly species contained similar proportions of host group-specific parasite lineages and thus do not appear to be associated with particular host groups.

Conclusions: The Leucocytozoon clade infecting thrushes, crows, and pigeons present in most represented blackfly species suggests a lack of association between hosts and blackflies, which can increase the probability of host switches of blood parasites. However, the composition of the simuliid species differed between nests of common buzzards, goshawks and red kites. This segregation can be explained by coinciding habitat preferences between host and vector, and may lead to the fast speciation of Leucocytozoon parasites. Thus, subtle ecological preferences and lack of host preference of vectors in the canopy may enable both parasite diversification and host switches, and enforce a habitat-dependent evolution of avian malaria parasites and related haemosporidia.
\end{abstract}

Keywords: Ornithophilic Simuliidae, Leucocytozoon, Host-specificity, Vector-driven speciation, Habitat choice, Canopy, Avian malaria

\section{Introduction}

Vector-transmitted parasites are extremely common but insufficiently understood [1]. In theory vectors can both catalyse and hamper the coevolution of hosts and

*Correspondence: nayden.chakarov@uni-bielefeld.de

${ }^{1}$ Department of Animal Behaviour, Bielefeld University, Bielefeld, Germany

Full list of author information is available at the end of the article symbionts but observations on the matter are scant [2]. Details of the vector behaviour are crucial for either process but also notoriously difficult to uncover.

Blood-feeding arthropods belong to the major ectoparasitic threats for wild vertebrates, not least because they transmit many potentially life-threatening disease agents [3]. Blackflies (Simuliidae), in particular, can be a major nuisance both due to mass outbreaks and the transmission of pathogens such as trypanosomes, 
haemosporidians and filarial nematodes [4]. Despite their key role in different environments, research on blackflies has for a long time focused on the accessible aquatic stages [5]. This has resulted in a knowledge gap on the feeding preferences of most species, only slowly improving in recent decades ([6], but see [7]). A substantial challenge to the exploration of the host specificity of most adult blackflies is their strong dependence on natural foraging conditions, which precludes most host choice experiments $[4,8]$. However, the development of molecular techniques allows the simultaneous identification of blackflies, the vertebrate blood parasites they contain and the species origin of the engorged blood. Nonetheless, for most simuliids, even of the most common and species-rich subgenera, feeding preferences are known only roughly. Most blackfly groups are so far classified only into mammalophilic or ornithophilic, which is mainly based on basic morphological features and the vertical distribution in the respective habitat $[4,9]$.

The vertical distribution of blackflies has generally been studied through capture by traps suspended at heights up to $10 \mathrm{~m}$ above ground and baited with $\mathrm{CO}_{2}$ or live galliform birds (presenting untypical hosts at such heights) $[10,11]$. However, in many forests, $10 \mathrm{~m}$ is under or the lowermost level of tree canopy, with potentially more than $20 \mathrm{~m}$ of canopy above remaining completely unexplored. In the present study, we aimed to identify the blackfly species attracted to birds of prey in their natural habitat, i.e. the upper canopy layers of a central European deciduous forest, as well as their blood diet and the parasitic lineages they carry. We therefore assumed an unorthodox approach and used free-living but stationary raptor broods as a natural bait with a long attraction period.

Simuliids are the principal vectors of avian haemosporidian parasites of the genus Leucocytozoon, which was recently shown to have the highest co-speciation rates and highest host-switching rates among blood parasites [12]. These characteristics of Leucocytozoon evolution may be assisted by the behaviour of blackflies [13]. The diversity and specialisation of Leucocytozoon lineages allow them to be used as natural markers for the diet of the respective vectors [14]. Identifying the genetic lineages of Leucocytozoon in blackflies can reveal the diet preferences and behaviour of separate vector species, which in turn can contribute to the understanding of Leucocytozoon transmission and evolution $[6,15]$.

Species of Leucocytozoon have been shown to be hostspecialised to a certain degree [13]. Within raptors, the sympatric genera of Accipiter (hawks) and Buteo (buzzards) are hosts of closely related but genetically distinct cryptic species of Leucocytozoon [16, 17]. This poses the question if the speciation of Leucocytozoon could have occurred in the same habitat due to different simuliid vectors feeding on the different raptor genera [7]. We therefore aimed to sample blackflies around broods of three of the most common birds of prey in central Europe, i.e. common buzzards Buteo buteo, northern goshawks Accipiter gentilis, and red kites Milvus milvus. We predicted that different Simulium species will be found around the nests of the three host species, corresponding to the transmitted parasites L. buteonis of buzzards and red kites, and L. mathisi of goshawks, thus providing an explanation for the high co-speciation rates of Leucocytozoon.

On the other hand, almost all species of Leucocytozoon have been shown to successfully develop in several tested species of blackflies [15]. Therefore, a potentially unselective prey choice by blackflies may occasionally lead to transmission of Leucocytozoon lineages to untypical hosts and facilitate host-switching. Under this scenario, we predicted that Leucocytozoon lineages transmitted by blackflies in the same habitat may have distantly related avian hosts.

\section{Methods}

\section{Study site and sample collection}

The study was performed between 2015 and 2017 in eastern Westphalia, Germany $\left(52.05^{\circ}-52.20^{\circ} \mathrm{N}\right.$ and $8.30^{\circ}-8.60^{\circ} \mathrm{E}$ ). The $300 \mathrm{~km}^{2}$ study area consists of a hilly landscape dominated by beech forest Fagus sylvatica, arable land, with smaller proportions of mixed and coniferous forest (Picea sp., Pinus sylvestris, Larix decidua) and meadows [18]. Forest patches are $0.001-7 \mathrm{~km}^{2}$ with a median size of $0.02 \mathrm{~km}^{2}$. Small streams intersect nearly every forest patch although many desiccate by July. Few permanent mid-sized streams also flow through the study area. Each study year, all forest patches in the area were searched for active nests of buzzards, red kites and goshawks. Such nests were regularly inspected until nestlings were ca. 3 weeks-old. Between 20th of May and 20th of June, the trees of active nests were climbed, and nestlings were brought to the ground for ringing [19, 20]. During the time when chicks were processed on the ground (20-30 $\mathrm{min})$, insects flying around the focal nest (10-30 m above ground) were caught with a scoop net. Blackflies were stored individually in 100\% ethanol.

\section{DNA extraction, amplification and sequencing}

DNA of single female blackflies was extracted from complete specimens using a standard phenol-chloroform protocol and quantified using a NanoDrop spectrophotometer (Thermo Fisher Scientific, Waltham, MA, USA). All samples were screened with three separate PCR assays: (i) blackfly species were determined with conserved primers targeting the cytochrome $c$ oxidase 
subunit 1 (cox1) DNA region (LCO1490: 5'-GGT CAA CAA ATC ATA AAG ATA TTG G- $3^{\prime}$ and HCO2198: 5'-TAA ACT TCA GGG TGA CCA AAA AAT CA-3') [21]; (ii) presence of vertebrate host DNA was tested with conserved primers targeting the vertebrate cytochrome $b$ (L14841: 5'-AAA AAG CTT CCA TCC AAC ATC TCA GCA TGA TGA AA- $3^{\prime}$ and H15149: $5^{\prime}$-AAA CTG CAG CCC CTC AGA ATG ATA TTT GTC CTC A-3') [22]; and (iii) presence of haemosporidian lineages within the blackfly individual was established with a nested PCR following the protocol of Perez-Martinez et al. [23], using the primer pair Plas1 (5'-GAG AAT TAT GGA GTG GAT GGT G-3') and HaemNR3 (5'-ATA GAA AGA TAA GAA ATA CCA TTC- $3^{\prime}$ ) for the first PCR and the internal primers 3760F ( $5^{\prime}$-GAG TGG ATG GTG TTT TAG AT- $3^{\prime}$ ) and Haem)R4 ( $5^{\prime}$-GAA ATA CCA TTC TGG AAC AAT ATG- $3^{\prime}$ ) for the second PCR. This nested PCR primer protocol amplifies the cytochrome $b$ gene of all haemosporidian genera, including raptor Leucocytozoon lineages which are less well detected with other nested PCR protocols [24]. Temperature profiles for the PCR reactions were according to [21-23]. PCR products were run on 2\% agarose gels. Amplicons were purified with ExoSAP (Thermo Fisher Scientific) and bi-directionally sequenced on an ABI 3730 Analyzer (Applied Biosystems, Waltham, MA, USA) with the BigDye Terminator v1.1 cycle sequencing kit (Thermo Fisher Scientific) using the respective two primers. Raw sequences were edited and aligned in Geneious 8.1.9 (www.geneious.com) and compared with sequences on GenBank, or in the case of 3760F/HaemJR4 with sequences on the MalAvi database, as of 28 January 2020 [24]. Phylogenetic Bayesian inference trees of blackfly and Leucocytozoon lineages were created with MrBayes with GTR+G model run for 100 000 generations burnin and 2 million generations postburnin and subsampled every 500 generations [25].

\section{Results}

We caught 156 blackflies from 64 raptor nests, with 1-14 blackfly individuals caught per nest. Of these 64 nests, 52 were common buzzard nests, 8 belonged to red kites and 4 to goshawks. Only 2 of the blackfly individuals were visibly engorged.

The sequencing of the cox 1 fragment of blackflies revealed 9 species: 86 individuals belonged to the Simulium (Nevermannia) vernum-group, including the species $S$. (N.) vernum, $S$. (N.) naturale, and $S$. (N.) cryophilum, which are indistinguishable based on the sequenced cox 1 fragment and will be further referred to as $S$. (N.) vernum*. All specimens were caught under 300 masl, which excludes the genetically very similar $S$. (N.) crenobium, appearing generally above 475 masl [26]. Ten blackfly individuals belonged to $S$. (N.) lundstromi. A further 35 blackflies were identified as $S$. (Eusimulium) angustipes and 18 as $S$. (E.) rubzovianum (formerly known as $S$. (E.) velutinum [27]). Furthermore, single individuals of the species $S$. (E.) aureum, $S$. (E.) petricolum, $S$. (N.) costatum, S. (Simulium) intermedium and S. (S.) posticatum were caught around the raptor nests. All specified blackflies had more than $98.5 \%$ sequence identity with reference sequences deposited on GenBank. Two specimens could not be genotyped.

The genotyping of vertebrate DNA retained in the blackflies, whether visibly engorged or not, revealed 6 blackfly individuals with vertebrate DNA, of which only 2 were visibly engorged. Three blood meal sources corresponded to common buzzard Buteo buteo: one $S$. lundstromi (carrying BUBT03, a Leucocytozoon-lineage typical of buzzards); one S. vernum* (carrying ASOT06, typical of owls); and one $S$. aureum (carrying COCOR12, typical of corvids). Another 2 individuals of S. vernum* contained DNA from red kite Milvus milvus (not carrying Leucocytozoon) and wood pigeon Columba palumbus (carrying EUSE02, a Leucocytozoon-lineage most closely related to parasites of thrushes), respectively. Furthermore, the only caught $S$. intermedium had fed on Bos taurus cattle, corresponding to its known mammalophilic diet [26, 28].

While the traces of blood and corresponding vertebrate DNA in genotyped blackflies provided a limited account of the host spectrum of high-foraging blackflies, the genotyping of parasite lineages delivered a much more comprehensive picture. From the 156 sampled flies, 46 (29.4\%) showed amplification of Leucocytozoon DNA, inferring that they had previously fed on infected bird hosts. Of these 46 individuals, 25 were identified as $S$. vernum*, 11 as S. angustipes, 5 as S. rubzovianum, 3 as S. lundstromi, 1 as S. petricolum, and 1 as $S$. aureum. The fraction of Leucocytozoon-carrying specimens was very similar for all analysed species at ca. $30 \%$.

We were able to identify single infections in 40 of the blackflies, which represented 19 lineages and revealed blackflies to carry parasites typical of thrushes, corvids, birds of prey, owls and tits, in this order of frequency (Table 1).

No Haemoproteus or Plasmodium lineages were detected in the analysed blackflies. Within the represented host groups, there was no obvious association between the identified simuliid taxa with any Leucocytozoon lineage or their corresponding host groups (Fig. 1).

The collected blackfly species showed the highest species diversity around nests of common buzzards. Simulium rubzovianum was disproportionately more common around nests of red kites, and S. vernum* was more common around nests of goshawks (Table 2, Chisquare test: $\left.\chi^{2}=45.6, d f=6, P<0.001\right)$. Nonetheless, 
no goshawk-specific Leucocytozoon lineages could be retrieved from any blackfly individual.

\section{Discussion}

Unorthodox approaches can yield unexpected findings about host-vector-parasite interactions. In this case, we aimed to discover whether closely related raptor species attract different simuliid species which may transmit distinct parasite lineages. In the process, we used natural hosts as bait, combined with manual netting. This approach helped to provide new knowledge about the diet of some of the most common European blackfly species. While most studies typically consider only engorged blackflies (but see $[7,14]$ ), we succeeded to derive more information on diets from analyses of the presence of Leucocytozoon-lineages in all specimens, combined with existent knowledge on their typical host groups.

\section{Blackfly species and host preference}

One of the main findings of our study was that certain blackfly species forage high up in the canopy and appear to be attracted to avian hosts. All but two out of the 154 identified individuals belonged to the subgenera $\mathrm{Nev}$ ermannia and Eusimulium, and nearly $30 \%$ of the individuals of both subgenera had fed on birds, as revealed by their Leucocytozoon load. This finding indicates that Nevermannia and Eusimulium are the dominant ornithophilic subgenera of the genus Simulium and as such being the most probable vectors of Leucocytozoon-lineages in central Europe (c.f. [13]). This corresponds to findings that other European species of Nevermannia attack thrushes and warblers, while blackflies of the $S$. (N.) vernum-group and $S$. (E.) aureum-group are relatively rare at heights under $10 \mathrm{~m}$ in spruce and pine forests $[9,13,28]$. Before this study, feeding preferences of Nevermannia and Eusimulium species were known only from $S$. (N.) silvestre, $S$. (N.) curvans, $S$. (E.) angustipes and $S$. (E.) aureum, the latter being one of the best examined ornithophilic blackflies and vectors of Leucocytozoon $[7,11,14,15,28,29]$. This species, however, appears to be rather rare in the upper canopy.

The composition of Leucocytozoon lineages found in this study shows that Nevermannia and Eusimulium species attack most avian host groups occurring in this canopy layer, which are sufficiently large and/or abundant, such as thrushes, corvids, pigeons, raptors, owls and tits. A similar size-and-abundance pattern of prey was found among ornithophilic and mammalophilic blackflies close to ground level in Scandinavia [28]. In contrast to the pattern found in Scandinavia, we did not find a strong association between blackfly species or haplotype and Leucocytozoon lineages, or their corresponding vertebrate host group [13]. In the study by Hellgren et al. [13], a limited number of engorged $S$. (N.) silvestre suggested a preference for thrushes. However, a much larger sample of $S$. (N.) silvestre from North America harboured Leucocytozoon lineages infecting avian species across the phylogeny, inferring that this species has a broad range of bird species in its diet [14]. This pattern corresponds much better to our findings from central Europe and supports the notion that species of Nevermannia and Eusimulium have habitat preferences but are otherwise indiscriminately ornithophilic (Fig. 1, Table 1). Our results deliver no information in which vector species the respective Leucocytozoon lineages can complete their development and life-cycle. However, experimental evidence suggests that most parasites of the genus Leucocytozoon are more restricted by the ecology of the vector than by its physiology [15].

The absence of non-Leucocytozoon parasites also provides insight into the behaviour of blackflies. Plasmodium and Haemoproteus are not transmitted and cannot fulfil their development in blackflies, but our protocol was apt to detect them, and their prevalence in the putative hosts is relatively high $[15,30]$. Therefore, their complete absence confirms that blackflies are not active in the foraging habitat after feeding and remain distant and inactive until the blood meal and potential abortive stages of Plasmodium and Haemoproteus are digested [4]. Individual blackflies possibly return after oviposition, as the period between two feedings has been measured to take 5-7 days in S. rugglesi [31].

\section{Vector behaviour}

To our knowledge, this is the first study of haemosporidian parasites being present in individual non-engorged blackflies. We found that nearly $30 \%$ of the blackfly individuals active in the upper canopy layers of central Europe are Leucocytozoon-carriers. Blackflies are likely to return to a large stationary food source such as a raptor brood after oviposition [31, 32]. Nonetheless, 30\% infected vectors are likely representative of the blackfly population in this habitat, since the raptor lineages potentially belonging to our "bait" accounted for only 15\% of all infected blackflies. Previous studies have either analysed pools of non-engorged blackflies or individual visibly engorged blackflies [11, 13, 14, 29]. Engorged blackflies are not active after feeding (0.1-0.3\% of all blackfly individuals caught in forests), but can account for up to $23.6 \%$ of all blackflies caught with a sweeping net in an alpine habitat [28, 33]. The frequency of Leucocytozoon-carriers among freshly engorged blackflies in Scandinavia was $62 \%$, being more representative of the Leucocytozoon prevalence in avian hosts, which is expected to be higher there than in central Europe $[13,15]$. On the other hand, close to $46 \%$ of pools of five non-engorged blackflies seem to contain 
Table 1 Leucocytozoon lineages molecularly identified in blackflies of different species, captured close to raptor nests and their respective typical vertebrate hosts

\begin{tabular}{|c|c|c|c|c|c|c|c|}
\hline \multirow[t]{2}{*}{ Leucocytozoon lineage } & \multicolumn{6}{|l|}{ Blackfly species } & \multirow{2}{*}{$\begin{array}{l}\text { Typical host clade } \\
\text { of Leucocytozoon } \\
\text { lineage }^{b}\end{array}$} \\
\hline & S. (N.) vernum*a & S. (N.) lundstromi & S. (E.) angustipes & $\begin{array}{l}\text { S. (E.) } \\
\text { rubzovianum }\end{array}$ & S. (E.) aureum & S. (E.) petricolum & \\
\hline MTUR2 & 1 & & & & & & Turdus \\
\hline NEVE01 & 4 & & & & & & Turdus \\
\hline STUR1 & 2 & & & 2 & & & Turdus \\
\hline EUSE2 & 5 & & & & & & Turdus (H143, 99\%) \\
\hline SANG $1^{C}$ & & & 1 & & & & Turdus (MTUR01, 95\%) \\
\hline SANG $2^{C}$ & 3 & & 1 & & & & Turdus (TUFAL01, 94\%) \\
\hline COCOR03 & 1 & & & & & & Corvidae, Columbidae \\
\hline COCORO9 & 1 & & & & & & Corvidae, Columbidae \\
\hline COCOR12 & & & & & 1 & & Corvidae, Columbidae \\
\hline COCOR13 & & 1 & 3 & 1 & & & Corvidae, Columbidae \\
\hline GAGLA06 & 1 & & & & & & Corvidae \\
\hline BUBT03 & & 1 & 1 & & & & Buteo \\
\hline BUTBUT03 & 3 & & & & & & Buteo \\
\hline MILANS04 & & & 2 & & & & Accipitridae \\
\hline ASOT06 & 1 & & 1 & & & & Strigidae \\
\hline STAL01 & & & & 1 & & & Strigidae \\
\hline PARUS18 & & & & 1 & & & Paridae \\
\hline PARUS20 & & & & & & 1 & Paridae \\
\hline $\begin{array}{l}\text { Unidentified/mixed } \\
\text { infection }\end{array}$ & 3 & 1 & 2 & & & & \\
\hline Total infected & 25 & 3 & 11 & 5 & 1 & 1 & \\
\hline Not infected & 61 & 7 & 24 & 13 & 0 & 0 & \\
\hline
\end{tabular}

a $S$. vernum, S. naturale and S. cryophilum are indistinguishable based on the sequenced cox 1 fragment and are treated together as $S$. $(N)$ vernum ${ }^{*}$

b Typical hosts of the respective Leucocytozoon lineage are derived from the MalAvi database [24]. Probable hosts of lineages known only from dipteran vectors are derived from BLAST matches. The genetically closest lineage with known vertebrate host and sequence similarity are indicated in parentheses

c Lineages described for the first time

Leucocytozoon lineages, suggesting a Leucocytozoon prevalence of approximately $20 \%$ in the corresponding blackfly populations $[11,14,29]$. Thus, we complement previous studies of Leucocytozoon-carrying blackflies with an individual-based estimate, which may be more precise but is specific to the upper canopy habitat of central Europe.

\section{Vector and host habitat choice}

Finally, we found a substantially different composition of blackfly species around the nests of three closely related avian hosts. Simulium (E.) rubzovianum was overrepresented around nests of red kites, and $S$. (N.) vernum* was the only blackfly species present around goshawk nests. At the same time, all species and the greatest diversity were represented around nests of common buzzards. This pattern could be due to host preference. Nests of the three raptor species can potentially be identifiable by odours, which is a primary sense for prey recognition outside of the visible range of blackflies [4, 34]. Buzzards for example, commonly have dead and decaying voles deposited around the nest. Red kites incorporate a great share of carrion and garbage in their nests and food, which lead to a distinct smell of the whole brood. Goshawks, on the other hand, feed mainly on birds and do not keep unconsumed prey remains at the nest. Although blackflies are not attracted to carrion per se, these compounds in addition to the native bird odours may enhance distinction of raptor species. However, it seems unlikely that the involved blackfly species discriminate against any of the raptor species, given the patterns outlined by the distribution of Leucocytozoon lineages found in this study.

Alternatively, the choice of breeding habitat by the three raptor species may predispose them to a different exposure of blackfly species around their nests. Red kites have a preference for open, dispersed deciduous and mixed forests, while goshawks prefer the core of bigger forests with a higher proportion of coniferous trees, and 


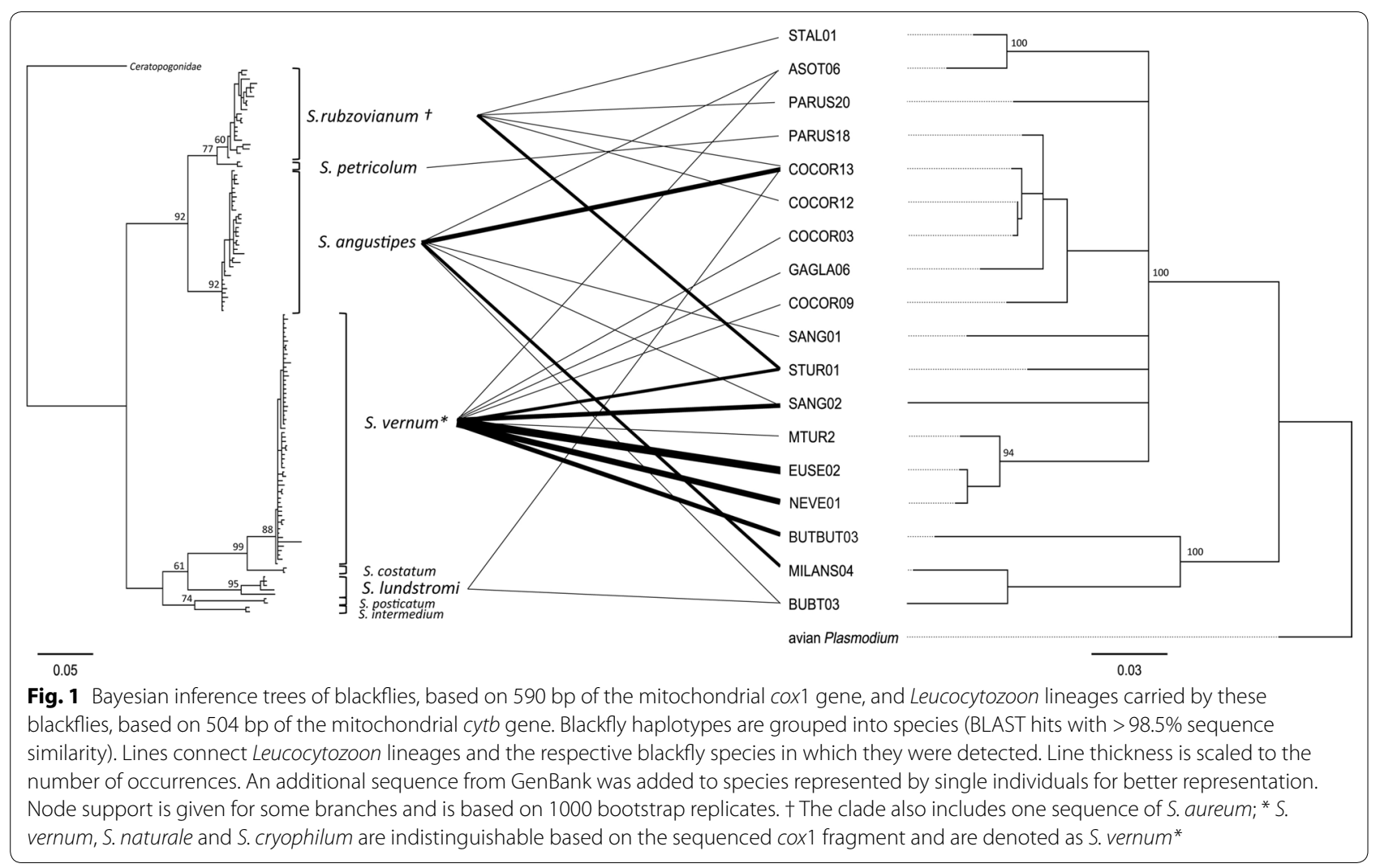

Table 2 Number of blackfly individuals of different species captured around the nests of three closely related sympatric raptor host species

\begin{tabular}{|c|c|c|c|c|}
\hline \multirow[t]{2}{*}{ Host species nest } & \multicolumn{4}{|l|}{ Blackfly species } \\
\hline & S. $(N$.$) vernum*a$ & S. (N.) lundstromi & S. (E.) angustipes & $\begin{array}{l}\text { S. }(\text { E.) } \\
\text { rubzovianum }\end{array}$ \\
\hline B. buteo (Common buzzard) & 68 & 10 & 34 & 8 \\
\hline M. milvus (Red kite) & 7 & & 1 & 10 \\
\hline A. gentilis (Northern goshawk) & 11 & & & \\
\hline
\end{tabular}

a S. vernum, S. naturale and S. cryophilum are indistinguishable based on the sequenced cox 1 fragment and are treated together as $S$. $(N)$ vernum*

Note: Additionally, single individuals of S. aureum, S. costatum, S. intermedium, and S. posticatum were caught around nests of common buzzards. One individual of S. petricolum was caught around a nest of red kites

buzzards cover the whole continuum from single trees to the core of big forests. These preferences may co-vary with the microhabitat foraging preferences of the different blackfly species, which are very poorly known [26]. Such a difference in the blackfly community, however, may have catalysed an ecological speciation of parasites, leading to the cryptic Leucocytozoon species infecting currently sympatric raptor species [16, 17, 35]. The substantial difference in sample size between the nests of the three raptor species does not allow us to adequately compare Leucocytozoon-lineage diversity around those nests and should be compensated by future studies.

\section{Conclusions}

Vectors are currently the least explored members of host-vector-parasite assemblages [6]. Knowledge of vector ecology and behaviour may be key to understanding the evolution, diversity, prevalence and health impact of parasite populations. Revealing the behaviour of minute arthropods remains challenging and beyond the capacity even of the current bio-logging revolution. Our study approached this aim by relying on the vast knowledge of associations between avian hosts and molecular lineages of blood parasites, which has accumulated in the last decades [24]. This allowed us to discover a distinct 
community of vectors, which seem to have similar hostseeking behaviour, while being active in the upper forest canopy. It thereby reaffirms the role of parasites as biological markers which can be useful to unveil details of vector biology [15].

\section{Acknowledgements}

We would like to thank Gediminas Valkiūnas, Institute of Ecology, Nature Research Centre, Vilnius, Lithuania, for initial encouragement to conduct this study. We are grateful for the indispensable help of Oliver Krüger, AnnaKatharina Mueller and Astrid Potiek from the Department of Animal Behaviour of Bielefeld University, Germany, during field work and of Prisca Viehoefer and Ann-Christin Polikeit, Bielefeld University, Germany, with sequencing.

\section{Authors' contributions}

NC gathered the samples and wrote the manuscript. HK, AW and NC performed the molecular analyses of the samples. DW performed morphological examinations of the samples. SB and NC analysed the data. SB and HK had major contributions to the writing of the manuscript. All authors read and approved the final manuscript.

\section{Funding}

During the duration of this study NC was funded by a Marie Curie grant (PIEFGA-2013-625883, H2020 Marie Skłodowska-Curie Actions) and a grant from Bielefeld University.

\section{Availability of data and materials}

All data supporting the conclusions of this article are included in the article.

\section{Ethics approval and consent to participate}

Field work at raptor nests was performed under the permit number 33.1942502-04-17/2514 issued by the regional authority LANUV in accordance with German federal law.

\section{Consent for publication}

Not applicable.

\section{Competing interests}

The authors declare that they have no competing interests.

\section{Author details}

${ }^{1}$ Department of Animal Behaviour, Bielefeld University, Bielefeld, Germany. ${ }^{2}$ Molecular Ecology and Evolution Lab, Department of Biology, Lund University, Lund, Sweden. ${ }^{3}$ Friedrich-Loeffler-Institut, Federal Research Institute for Animal Health, Greifswald, Insel Riems, Germany. ${ }^{4}$ Research Area 2 'Land Use and Governance', Leibniz Centre for Agricultural Landscape Research, Muencheberg, Germany.

Received: 1 February 2020 Accepted: 8 June 2020

Published online: 15 June 2020

\section{References}

1. Schmid-Hempel P. Evolutoinary parasitology. The integrated study of infections, immunology, ecology, and genetics. Oxford: Oxford University Press; 2011.

2. Ricklefs RE, Fallon SM, Bermingham E. Evolutionary relationships, cospeciation, and host switching in avian malaria parasites. Syst Biol. 2004;53:111-9.

3. Lehane MJ. The biology of blood-sucking in insects. Cambridge: Cambridge University Press; 2005.

4. Crosskey RW. The natural history of blackflies. Chichester: Wiley; 1990

5. Malmqvist B, Adler PH, Kuusela K, Merritt RW, Wotton RS. Black flies in the boreal biome, key organisms in both terrestrial and aquatic environments: a review. Ecoscience. 2004;11:187-200.

6. Santiago-Alarcon D, Palinauskas V, Schaefer HM. Diptera vectors of avian Haemosporidian parasites: untangling parasite life cycles and their taxonomy. Biol Rev. 2012;87:928-64.
7. Bennett GF. On some ornithophilic blood-sucking diptera in Algonquin Park, Ontario, Canada. Can J Zool. 1960;38:377-89.

8. Lowther J, Wood D. Specificity of a black fly, Simulium euryadminiculum Davies, toward its host, the common loon. Can Entomol. 1964;96:911-3.

9. Swanson D, Adler P, Malmqvist B. Spatial stratification of host-seeking Diptera in boreal forests of northern Europe. Med Vet Entomol. 2012;26:56-62.

10. Malmqvist B, Strasevicius D, Adler PH. Catches of bloodsucking blackflies (Diptera: Simuliidae) tell different stories depending on sampling method. Entomol Fennica. 2007:18:110-6.

11. Synek P, Munclinger P, Albrecht T, Votypka J. Avian haemosporidians in haematophagous insects in the Czech Republic. Parasitol Res. 2013;112:839-45.

12. Alcala $N$, Jenkins $T$, Christe $P$, Vuilleumier $S$. Host shift and cospeciation rate estimation from co-phylogenies. Ecol Lett. 2017;20:1014-24.

13. Hellgren $O$, Bensch $S$, Malmqvist B. Bird hosts, blood parasites and their vectors-associations uncovered by molecular analyses of blackfly blood meals. Mol Ecol. 2008;17:1605-13.

14. Murdock CC, Adler PH, Frank J, Perkins SL. Molecular analyses on hostseeking black flies (Diptera: Simuliidae) reveal a diverse assemblage of Leucocytozoon (Apicomplexa: Haemospororida) parasites in an alpine ecosystem. Parasit Vectors. 2015;8:343.

15. Valkiūnas G. Avian malarial parasites and other Haemosporidia. Boca Raton: CRC Press; 2005

16. Valkiūnas G, Sehgal RNM, lezhova TA, Hull AC. Identification of Leucocytozoon toddi group (Haemosporida: Leucocytozoidae), with remarks on the species taxonomy of leucocytozoids. J Parasitol. 2010;96:170-7.

17. Sehgal RNM, Hull AC, Anderson NL, Valkiūnas G, Markovets MJ, Kawamura $\mathrm{S}$, et al. Evidence for cryptic speciation of Leucocytozoon spp. (Haemosporida, Leucocytozoidae) in diurnal raptors. J Parasitol. 2006:92:375-9.

18. Mueller A-K, Chakarov N, Heseker H, Krüger O. Intraguild predation leads to cascading effects on habitat choice, behaviour and reproductive performance. J Anim Ecol. 2016;85:774-84.

19. Chakarov N, Pauli M, Mueller AK, Potiek A, Grunkorn T, Dijkstra C, et al. Territory quality and plumage morph predict offspring sex ratio variation in a raptor. PLoS ONE. 2015;10:e0138295.

20. Chakarov N, Boerner M, Krüger O. Fitness in common buzzards at the cross-point of opposite melanin-parasite interactions. Funct Ecol. 2008:22:1062-9.

21. Folmer O, Black W, Lutz R, Vrijenhoek R. DNA primers for amplification of mitochondrial cytochrome c oxidase subunit I from diverse metazoan invertebrates. Mol Mar Biol Biotechnol. 1994;3:294-9.

22. Kocher TD, Thomas WK, Meyer A, Edwards SV, Pääbo S, Villablanca FX, et al. Dynamics of mitochondrial DNA evolution in animals: amplification and sequencing with conserved primers. Proc Natl Acad Sci USA. 1989;86:6196-200.

23. Pérez-Rodríguez A, de la Puente J, Onrubia A, Pérez-Tris J. Molecular characterization of haemosporidian parasites from kites of the genus Milvus (Aves: Accipitridae). Int J Parasitol. 2013;43:381-7

24. Bensch S, Hellgren O, Pérez-Tris J. MalAvi: a public database of malaria parasites and related haemosporidians in avian hosts based on mitochondrial cytochrome $b$ lineages. Mol Ecol Resour. 2009;9:1353-8.

25. Ronquist F, Huelsenbeck JP. MrBayes 3: Bayesian phylogenetic inference under mixed models. Bioinformatics. 2003:19:1572-4.

26. Lechthaler W, Car M. Simuliidae: key to larvae and pupae from central and western Europe. Vienna: eutaxa-Technisches Büro für Biologie; 2005.

27. Adler P, Cherairia M, Arigue S, Samraoui B, Belqat B. Cryptic biodiversity in the cytogenome of bird-biting blackflies in North Africa. Med Vet Entomol. 2015:29:276-89.

28. Malmqvist B, Strasevicius D, Hellgren O, Adler PH, Bensch S. Vertebrate host specificity of wild-caught blackflies revealed by mitochondrial DNA in blood. Proc R Soc Lond Ser B Biol Sci. 2004;271:S152-5.

29. Synek P, Popelková A, Koubínová D, Štastný K, Langrová I, Votýpka $J$, et al. Haemosporidian infections in the Tengmalm's owl (Aegolius funereus) and potential insect vectors of their transmission. Parasitol Res. 2016;115:291-8.

30. Scaglione FE, Cannizzo FT, Pregel P, Perez-Rodriguez AD, Bollo E. Blood parasites in hooded crows (Corvus corone cornix) in northwest Italy. Vet Ital. 2016;52:111-6.

31. Bennett GF. Use of $P^{32}$ in the study of a population of Simulium rugglesi (Diptera: Simuliidae) in Algonquin Park, Ontario. Can J Zool. 1963:41:831-40. 
32. Chakarov N, Linke B, Boerner M, Goesmann A, Kruger O, Hoffman I. Apparent vector-mediated parent-to-offspring transmission in an avian malaria-like parasite. Mol Ecol. 2015;24:1355-63.

33. Imura T, Sato Y, Ejiri H, Tamada A, Isawa H, Sawabe K, et al. Molecular identification of blood source animals from black flies (Diptera: Simuliidae) collected in the alpine regions of Japan. Parasitol Res. 2010;106:543-7.

34. Bennett GF, Fallis AM, Campbell AG. The response of Simulium (Eusimulium) euryadminiculum Davies (Diptera: Simuliidae) to some olfactory and visual stimuli. Can J Zool. 1972;50:793-800.
35. McCoy KD. Sympatric speciation in parasites — what is sympatry? Trends Parasitol. 2003;19:400-4.

\section{Publisher's Note}

Springer Nature remains neutral with regard to jurisdictional claims in published maps and institutional affiliations.
Ready to submit your research? Choose BMC and benefit from:

- fast, convenient online submission

- thorough peer review by experienced researchers in your field

- rapid publication on acceptance

- support for research data, including large and complex data types

- gold Open Access which fosters wider collaboration and increased citations

- maximum visibility for your research: over 100M website views per year

At BMC, research is always in progress.

Learn more biomedcentral.com/submissions 